\title{
c-Myc maintains the self-renewal and chemoresistance properties of colon cancer stem cells
}

\author{
HUAN-LE ZHANG ${ }^{1}$, PING WANG ${ }^{2}$, MIAO-ZHEN LU ${ }^{1}$, SAN-DIAN ZHANG ${ }^{1}$ and LU ZHENG ${ }^{1}$ \\ ${ }^{1}$ Department of Oncology, Lihuili Hospital of Ningbo Medical Center; ${ }^{2}$ Department of Molecular Biology, \\ Ningbo University School of Medicine, Ningbo, Zhejiang 315000, P.R. China
}

Received January 10, 2016; Accepted December 18, 2018

DOI: $10.3892 / \mathrm{ol} .2019 .10081$

\begin{abstract}
Cancer stem cells (CSCs) are responsible for cancer formation, recurrence and drug resistance. c-Myc, one of the core markers for stem cells, has recently been considered to serve as a link between malignancy and 'stemness'. However, the precise function of c-Myc in colon CSCs is still unclear. In the present study, a subpopulation of colon CSCs expressing a CD133 surface phenotype was isolated from the human HT-29 cell line, which possess greater tumor sphere-forming efficiency and have higher expression of 'stemness'-associated genes compared with CD133-negative cells. Furthermore, it was demonstrated that c-Myc was highly expressed in $\mathrm{CD} 133^{+}$ colon CSCs. Knockdown of c-Myc expression with small interfering RNA in colon CSCs can significantly inhibit tumor sphere formation, reduce the invasive and migratory capacity of $\mathrm{CD}_{133^{+}}$cells in vitro, and suppress the tumorigenicity of colon CSCs in vivo. In addition, it was suggested that c-Myc silencing may sensitize colon CSCs to chemotherapy-induced cytotoxicity via the downregulation of ABCG2 and ABCB5. These findings support a central role for c-Myc in maintaining the self-renewing and chemoresistant properties of colon CSCs.
\end{abstract}

\section{Introduction}

Colon cancer was the third leading cause of malignancy-associated deaths in America in 2014, and represents a major therapeutic challenge (1). Despite advances in therapy, including surgery and chemotherapy, tumor recurrence and metastasis cannot yet be effectively prevented (2). Accumulating evidence suggests the presence of a small subpopulation of cells, known as cancer stem cells (CSCs), in colon cancer that exhibit stem-like features, and promote tumor formation, metastasis and resistance to therapy (3-6). Therefore, therapies targeting

Correspondence to: Dr Huan-Le Zhang, Department of Oncology, Lihuili Hospital of Ningbo Medical Center, 57 Xingning Road, Ningbo, Zhejiang 315000, P.R. China

E-mail: zh1520@163.com

Key words: c-Myc, cancer stem cells, self-renewal, chemoresistance colon CSCs may be a promising approach to eradicating colon cancer (7-9).

Higher expression of c-Myc has been found in colon cancer cells, relative to in normal mucosa, suggesting that c-Myc is involved in tumor development and progression (10). Recently, c-Myc has been recognized as an important regulator of stem cell biology (11); thus, it may serve as a link between malignancy and 'stemness'. Previous studies have shown that c-Myc has a key role in regulating the self-renewal and survival of glioma CSCs, and targeting c-Myc pathways may significantly improve brain tumor therapies (12). It is also reported that c-Myc can contribute to the multidrug resistance profile by dysregulating the transcription of specific $\mathrm{ABC}$ transporter genes in cancer cells $(13,14)$. However, c-Myc gene regulation mechanisms underlying the maintenance of self-renewal and drug-resistant properties in colon CSCs are still unclear.

In a previous study (6), a subpopulation of colon CSCs expressing a CD133 surface phenotype were isolated from the human HT-29 colonic adenocarcinoma cell line using flow cytometry cell sorting (FACS). CD133+ cells possess a greater tumor sphere-forming efficiency in vitro and higher tumorigenic potential in vivo. Furthermore, $\mathrm{CD} 133^{+}$cells are endowed with stem cell-like properties, including the expression of 'stemness' genes, such as Wnt2, BMI1, Oct3/4, Notch1 and c-Myc, and the maintenance of self-renewal and differentiation capacities. The miRNA expression signature of colon CSCs was revealed in our previous study (6).

In the present study, it was further demonstrated that c-Myc was consistently overexpressed in colon CSCs. Additionally, it was shown that downregulation of c-Myc suppressed the self-renewal of colon CSCs and the growth of xenografts. Furthermore, it was suggested that the depletion of c-Myc may enhance the chemosensitivity of colon CSCs through the downregulation of ABCG2 and ABCB5 expression. The present study demonstrated that the expression of c-Myc has a crucial role in maintaining the self-renewal and chemoresistance of colon CSCs.

\section{Materials and methods}

Cell culture and colon sphere formation. The human HT-29 colonic adenocarcinoma cell line was obtained from the American Type Culture Collection (ATCC; Manassas, VA, USA) and was maintained in McCoy's 5A medium 
(Gibco; Thermo Fisher Scientific, Inc., Waltham, MA, USA) containing $10 \%$ fetal bovine serum (FBS; Gibco; Thermo Fisher Scientific, Inc.) at $37^{\circ} \mathrm{C}$ and $5 \% \mathrm{CO}_{2}$, and the medium was changed every 3 days. Cells were passaged at $80 \%$ confluence and seeded at $20 \%$ confluence to keep them at optimal proliferating conditions.

For colon sphere formation, single-cell suspensions were cultured in a DMEM/F-12 basal serum-free medium (Gibco; Thermo Fisher Scientific, Inc.), containing $2 \mathrm{mM}$ L-glutamine, $1 \mathrm{mg} / \mathrm{ml} \mathrm{NaHCO} 3,4 \mu \mathrm{g} / \mathrm{ml}$ heparin, $100 \mu \mathrm{g} / \mathrm{ml}$ transferrin, $25 \mu \mathrm{g} / \mathrm{ml}$ insulin, $30 \mathrm{nM}$ sodium selenite anhydrous and $20 \mathrm{nM}$ progesterone (Sigma-Aldrich; Merck KGaA, Darmstadt, Germany), and supplemented with $20 \mathrm{ng} / \mathrm{ml}$ pro-epidermal growth factor (EGF; R\&D Systems China Co., Ltd., Shanghai, China) and $10 \mathrm{ng} / \mathrm{ml}$ fibroblast growth factor 2 (FGF-2; R\&D Systems China Co.).

In chemoresistance experiments, colon CSCs and adherent HT-29 cells were exposed to $50 \mu \mathrm{M}$ 5-FU (Sigma-Aldrich; Merck KGaA) or $1.25 \mu \mathrm{M}$ oxaliplatin (Sigma-Aldrich; Merck KGaA) or FOLFOX (50 $\mu \mathrm{M}$ 5-FU plus $1.25 \mu \mathrm{M}$ oxaliplatin) for $72 \mathrm{~h}$, and then observed by inverted phase-contrast microscope.

FACS. For the isolation of $\mathrm{CD} 133^{+}$and $\mathrm{CD} 133^{-}$populations, single-cell suspensions were incubated with phycoerythrin (PE)-conjugated anti-human CD133/1 (1:1,000; 130-108, AC133 clone; Miltenyi Biotec GmbH, Bergisch Gladbach, Germany) and FcR blocking reagent (Miltenyi Biotec $\mathrm{GmbH}$ ) in staining solution containing 1\% BSA and $2 \mathrm{mM}$ EDTA for $10 \mathrm{~min}$ at $4^{\circ} \mathrm{C}$. Isotype-matched mouse immunoglobulin served as the control. Samples were analyzed and sorted with a FACSAria flow cytometer (BD Biosciences, Franklin Lakes, NJ, USA). For the positive and negative populations, only the top $10 \%$ most brightly stained cells or the bottom $10 \%$ most dimly stained cells were selected, respectively. Following cytofluorimetric sorting, viability was assessed using trypan blue exclusion; cell purity was controlled via flow cytometry with an antibody against CD133/2-phycoerythrin (1:1,000; 130-090-853, 293C3 clone; Miltenyi Biotec GmbH) in staining solution containing $1 \%$ BSA and $2 \mathrm{mM}$ EDTA for $10 \mathrm{~min}$ at $4^{\circ} \mathrm{C}$.

Small interfering RNA (siRNA) transfection. siRNA specific to c-Myc (sc-29227; sense, 5'-CAGAAAUGUCCUGAGCAA UUU-3' and antisense, 5'-AUUGCUCAGGACAUUUCU GUU-3') and a scrambled siRNA (sc-37007; sense, 5'-GAC UUCAUAAGGCGCAUGCUU-3' and antisense, 5'-GCAUGC GCCUUAUGAAGUCUU-3') were obtained from Santa Cruz Biotechnology, Inc. (Dallas, TX, USA). Sorted CD133 ${ }^{+}$HT-29 cells $\left(5 \times 10^{5}\right)$ were seeded into 6-well plates in SFM. After $24 \mathrm{~h}$, siRNA was transfected into $\mathrm{CD} 133^{+}$cells at a final concentration of $100 \mathrm{nM}$ using Lipofectamine ${ }^{\mathrm{TM}}$ RNAiMAX reagent (Invitrogen; Thermo Fisher Scientific, Inc.), according to the manufacturer's instructions. The cells were collected for a series of experiments at $48 \mathrm{~h}$ after transfection.

In vivo tumorigenicity. Transfected cells $\left(5 \times 10^{5}\right)$ were resuspended in $50 \mu \mathrm{l}$ PBS, and cell aliquots were diluted 1:1 with growth factor-reduced Matrigel matrix (BD Biosciences) before injection. A total of $10 \mathrm{BALB} / \mathrm{c}$ nude female mice
(6 weeks old), weighing 18-22 g, were purchased from the Shanghai Laboratory Animal Center of the Chinese Academy of Sciences (Shanghai, China). The mice were maintained under a $12 \mathrm{~h} \mathrm{light/dark} \mathrm{cycle} \mathrm{at} \mathrm{an} \mathrm{ambient} \mathrm{temperature} \mathrm{of}$ $24 \pm 1^{\circ} \mathrm{C}$ in a humidity-controlled environment in a specific pathogen-free animal facility at the Center for Animal Experimentation, Medical Institute of Shanghai Jiao Tong University (Shanghai, China). Sterilized water and $\gamma$-irradiated diet were provided to animals ad libitum. Mice were subcutaneously injected with post-transfected cells, and the mice were observed every 2 weeks. The duration of the experiments was 8 weeks following transplantation, after which all mice were anesthetized with $1 \%$ pentobarbital sodium $(40 \mathrm{mg} / \mathrm{kg})$ and euthanized by cervical dislocation. Every mouse had one tumor, which was excised and measured. Each group consisted of 5 mice.

Western blotting. Protein was extracted with protein extraction kit (Minute $^{\mathrm{TM}}$ Protein Extraction Kit; SD-001; Invent Biotechnologies, Inc., Plymouth, MN, USA), and the protein concentration was detected using the bicinchoninic acid (BCA) Protein Assay kit (Beyotime Institute of Biotechnology, Haimen, China). Subsequently, $20 \mu \mathrm{g}$ protein was separated via $10 \%$ SDS-PAGE, transferred onto a polyvinylidene fluoride membrane, and blocked in 5\% skimmed milk at $20^{\circ} \mathrm{C}$ for $1 \mathrm{~h}$. The following primary antibodies were added to membranes at a dilution of $1: 1,000$ at $4^{\circ} \mathrm{C}$ for $24 \mathrm{~h}$ : Bmil (sc-13519; Santa Cruz Biotechnology, Inc.), ABCG2 (sc-13519; Santa Cruz Biotechnology, Inc.), ABCB5 (sc-517565; Santa Cruz Biotechnology, Inc.), Sox2 (sc-365964; Santa Cruz Biotechnology,Inc.), Wnt2 (sc-5208; Santa Cruz Biotechnology, Inc.), Oct3/4 (sc-5279; Santa Cruz Biotechnology, Inc.), CD133 (130-108-062, AC133 clone; Miltenyi Biotec GmbH), c-Myc (ab32072; Abcam, Cambridge, MA, USA), $\beta$-actin (ab1801; Abcam) and GAPDH (ab8245; Abcam). The membrane was then incubated with a horseradish peroxidase-labeled secondary antibody $(1: 2,000$; A0216; Beyotime Institute of Biotechnology) at room temperature for $1 \mathrm{~h}$. The membrane was visualized using an enhanced chemiluminescence detection system (Bio-Rad Laboratories, Inc., Hercules, CA, USA), and analyzed using a gel analyzer (Bio-Rad Laboratories, Inc.).

Chemo-sensitivity assays. Chemosensitivity assays were conducted using a Cell Counting Kit-8 (CCK-8; Dojindo Laboratories, Kumamoto, Japan). Sorted CD133 ${ }^{+}$HT-29 cells were seeded into 96 -well plates at a density of $4 \times 10^{3}$ cells/well in $100 \mu \mathrm{l} \mathrm{SFM}$ overnight, and then transfected with $100 \mu \mathrm{M}$ of an indicated siRNA. For the chemosensitivity assay, post-transfected $(48 \mathrm{~h})$ cells were exposed to 5 -FU $(50 \mu \mathrm{M})$, oxaliplatin $(1.25 \mu \mathrm{M})$ and FOLFOX $(50 \mu \mathrm{M} 5$-FU plus $1.25 \mu \mathrm{M}$ oxaliplatin). After incubation for $72 \mathrm{~h}$, a CCK-8 assay was performed, and the survival rate of cells was calculated as follows: OD treatment/OD control x 100. Experiments were performed in triplicate.

Migration and invasion assays. Migration and invasion assays were performed in 24-well Transwell chambers with 8-mm pore polycarbonate filter inserts (Corning Inc., Corning, NY, USA). A total of $5 \times 10^{4}$ post-transfected cells were seeded on uncoated or Matrigel-coated inserts in $500 \mu 1$ serum-free 
A

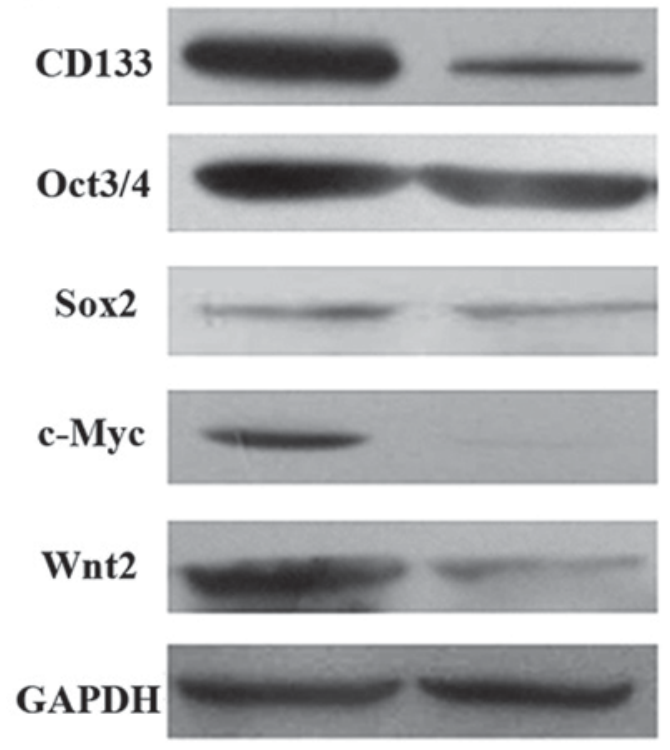

B

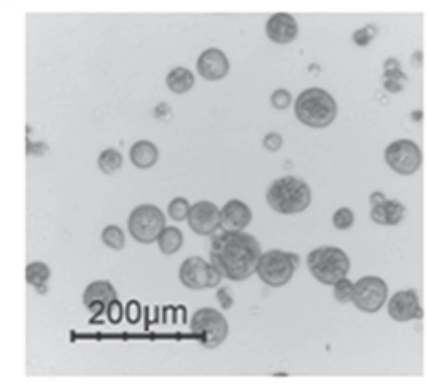

C

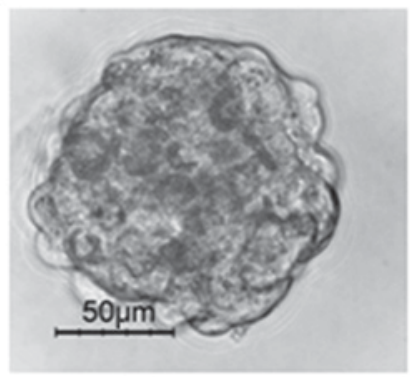

Figure 1. Isolated CD133+ HT-29 cells exhibit stem-like features in vitro. (A) 'Stemness' genes, c-Myc, Sox2, Oct4, CD133 and Wnt2, were highly expressed in $\mathrm{CD}_{133^{+}}$cells, as assessed by western blotting; lane 1, CD133+ cells; lane 2, CD133- cells. (B and C) CD133 colon cancer cells were expanded in vitro as undifferentiated spheres in serum-free medium, as observed by inverted phase contrast microscopy after 2 weeks of culture.

medium for migration or invasion assays, respectively. The lower chambers were filled with $500 \mu 110 \%$ FBS-supplemented DMEM/F12 medium. After $48 \mathrm{~h}$, cells on the upper side of the filter were removed, and the cells on the lower surface of the insert were fixed with $4 \%$ paraformaldehyde $(500 \mu \mathrm{l})$ at room temperature for $20 \mathrm{~min}$, washed twice with PBS $(500 \mu \mathrm{l})$ and stained with crystal violet $(400 \mu \mathrm{l})$ at $37^{\circ} \mathrm{C}$ for $20 \mathrm{~min}$. The number of stained cells was counted in 3 randomly selected fields of view under a light microscope using a x10 objective (Nikon Corporation, Tokyo, Japan). Assays were performed in triplicate.

Statistical analysis. SPSS 19.00 software (IBM Corp., Armonk, NY, USA) was used to analyze the data in the present study. Data are presented as the mean \pm standard deviation and differences between 2 groups were compared using Student's t-test. $\mathrm{P}<0.05$ was considered to indicate a statistically significant difference.

\section{Results}

$\mathrm{CD}_{133^{+}}$cells exhibit stem-likefeatures in vitro.It was previously proven that CD133 is a powerful marker for detecting colon CSC subpopulations in the human HT-29 colonic adenocarcinoma cell line (6). In the present study, CD133+ and CD133- cells were purified from the HT-29 cell line using FACS. To explore the molecular features of colon CSCs, the expression of genes involved in stem cell-associated pathways was detected by western blotting. As shown in Fig. 1A, the expression of 'stemness' genes, such as c-Myc, Sox2, Oct4, CD133 and Wnt2, was higher in $\mathrm{CD}_{133^{+}}$cells compared with that in CD133- cells. As has been reported previously (6), isolated $\mathrm{CD} 133^{+}$cells were able to generate tumor spheres when cultured for 10-14 days in serum-free stem cell medium supplemented with $20 \mathrm{ng} / \mathrm{ml}$ EGF and $10 \mathrm{ng} / \mathrm{ml}$ FGF-2. Floating sphere-like cellular aggregates were formed, as shown in Fig. 1B and C. Additionally, colon cancer spheres had a larger volume and more compact sphere structure following the third cell-culture passage (data not shown). The results demonstrated that CD133+ HT-29 cells exhibit stem-like properties, including the expression of 'stemness' genes and self-renewal capacity.

Depletion of c-Myc attenuates tumor sphere formation among colon CSCs. Tumor sphere generation in vitro is indicative of self-renewal potential $(5,9)$. To investigate whether c-Myc regulates the self-renewal capacity of colon CSCs, c-Myc expression was downregulated in $\mathrm{CD} 133^{+}$colon CSCs using siRNA, and the results showed that c-Myc-siRNA cells formed smaller and fewer tumor spheres than the scramble-siRNA control and $\mathrm{CD}_{133^{+}}$counterparts when cultured in serum-free stem cell medium (Fig. 2A). On the other hand, the c-Myc-siRNA group demonstrated lower Bmil expression levels. These data reveal that c-Myc serves an important role in regulating the self-renewal ability of colon CSCs cells, partly through regulating Bmi1.

Knockdown of $c-M y c$ inhibits the invasion and migration potential of colon CSCs. Invasive and migratory abilities are critical features of CSCs; thus, it was examined whether c-Myc affected these features in colon cancer. Here, in vitro assays were conducted to evaluate migratory and invasive capacities. A Transwell assay found that c-Myc-siRNA cells display a significant decrease in cell motility relative to their scramble-siRNA counterparts $(\mathrm{P}<0.01$, Fig. 2B). Additionally, significantly fewer c-Myc-siRNA cells were able to invade Matrigel-coated inserts in the Transwell migration chambers 
A

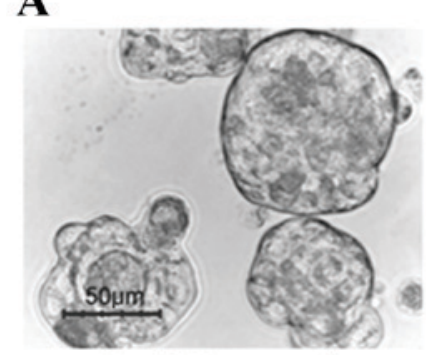

$\mathrm{CD} 133^{+}$control

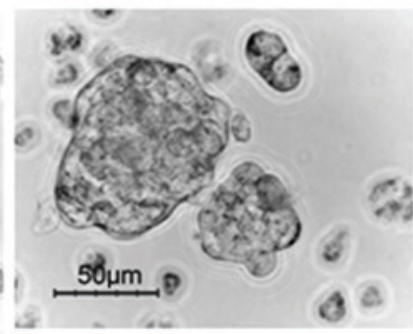

Si-scramble

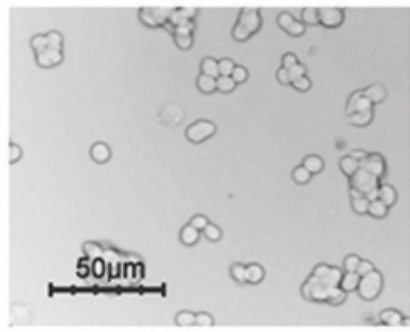

Si-Myc
B

C
Invasion

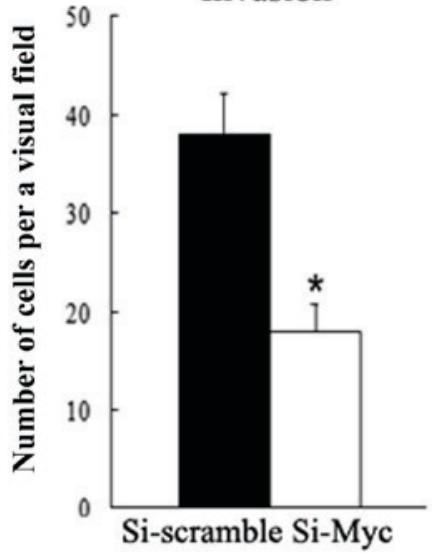

Migration

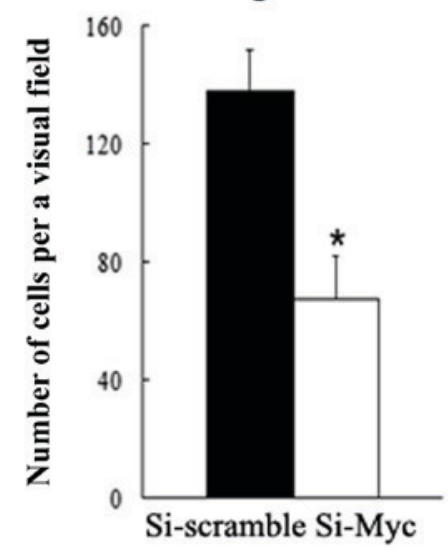

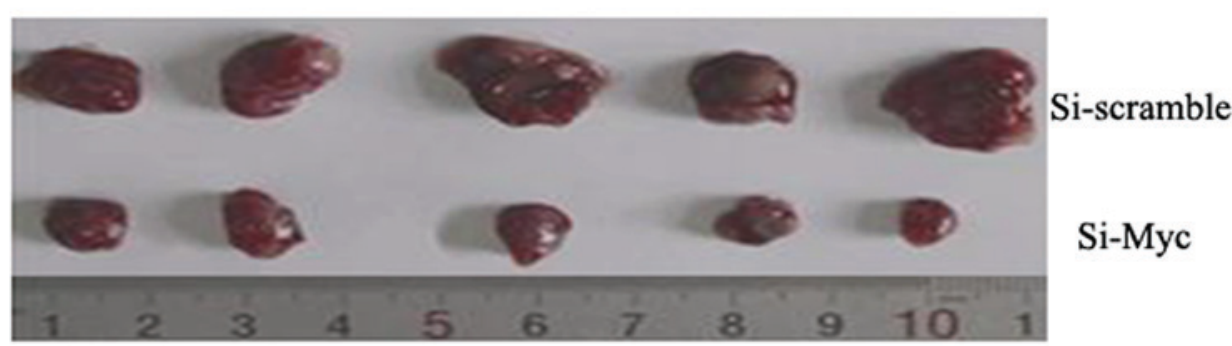

Figure 2. Knockdown of c-Myc in colon CSCs attenuates sphere formation and cell mobility in vitro, and inhibits tumorigenicity in vivo. (A) c-Myc-siRNA was transfected into CD133+ cells, which formed smaller and fewer tumor spheres than the scramble-siRNA control and CD133 ${ }^{+}$counterpart groups cultured in serum-free stem cell medium. Representative images of colon cell spheres observed by the inverted phase contrast microscope are shown (bars, $50 \mu \mathrm{m}$ ). (B) Knockdown of c-Myc inhibits the invasion and migration potential of CD133+ cells (all assays were performed in triplicate; data are presented as the mean \pm standard deviation; ${ }^{P} \mathrm{P}<0.05$, compared with the control). (C) c-Myc-siRNA-transfected CD133 ${ }^{+}$cells generated smaller xenograft tumors when compared with the scramble-siRNA group; the grafts were excised at 8 weeks after transplantation in vivo. siRNA, small interfering RNA; CSC, cancer stem cell.

than with the $\mathrm{NC}$ counterparts $(\mathrm{P}<0.01$, Fig. 2B). These results indicate that knockdown of c-Myc inhibits the invasion and migration potential of colon CSCs, promoting a functional phenotype associated with tumor aggressiveness.

c-Myc-siRNA suppresses the tumorigenicity of colon CSCs in vivo. To assess the function of $\mathrm{c}-\mathrm{Myc}$ with respect to tumorigenicity in vivo, tumor development experiments were carried out. Nude mice were subcutaneously injected with c-Myc-siRNA transfected CD133+ cells or scramble-siRNA counterparts. As shown in Fig. 2C, mice in the c-Myc-siRNA group developed much smaller subcutaneous tumors than those in the scramble-siRNA group. The tumor diameters of the c-Myc-siRNA group and the scramble-siRNA group were $1.07 \pm 0.18$ and $0.43 \pm 0.13 \mathrm{~cm}$, respectively. In vivo experiment results illustrated that c-Myc-siRNA attenuates the tumorigenicity of $\mathrm{CD}_{133^{+}}$colon CSCs.
Depletion of $c-M y c$ enhances the chemosensitivity in colon $C S C$ s through the downregulation of $A B C G 2$ and $A B C B 5$ expression. Previous studies have reported that CSCs are widely resistant to chemotherapeutic drugs $(7,8)$. Herein, colon CSCs and adherent cells were exposed to 5-FU $(50 \mu \mathrm{M})$ or oxaliplatin $(1.25 \mu \mathrm{M})$ or FOLFOX $(50 \mu \mathrm{M} 5$-FU plus $1.25 \mu \mathrm{M}$ oxaliplatin) for $72 \mathrm{~h}$; as expected, the chemotherapy of HT-29 adherent cells resulted in a significant increase in cell death and disintegration compared with colon CSCs, as observed via inverted phase-contrast microscopy (Fig. 3A). Furthermore, to evaluate the effect of c-Myc on the drug resistance of colon CSCs, a chemosensitivity assay was conducted. Transfected cells were treated with the same chemotherapy strategy as aforementioned. After incubation for further $72 \mathrm{~h}$, the CCK-8 assay results demonstrated that the survival rates of c-Myc-siRNA-transfected cells were significantly reduced compared with those of the scramble-siRNA group (Fig. 3B). 


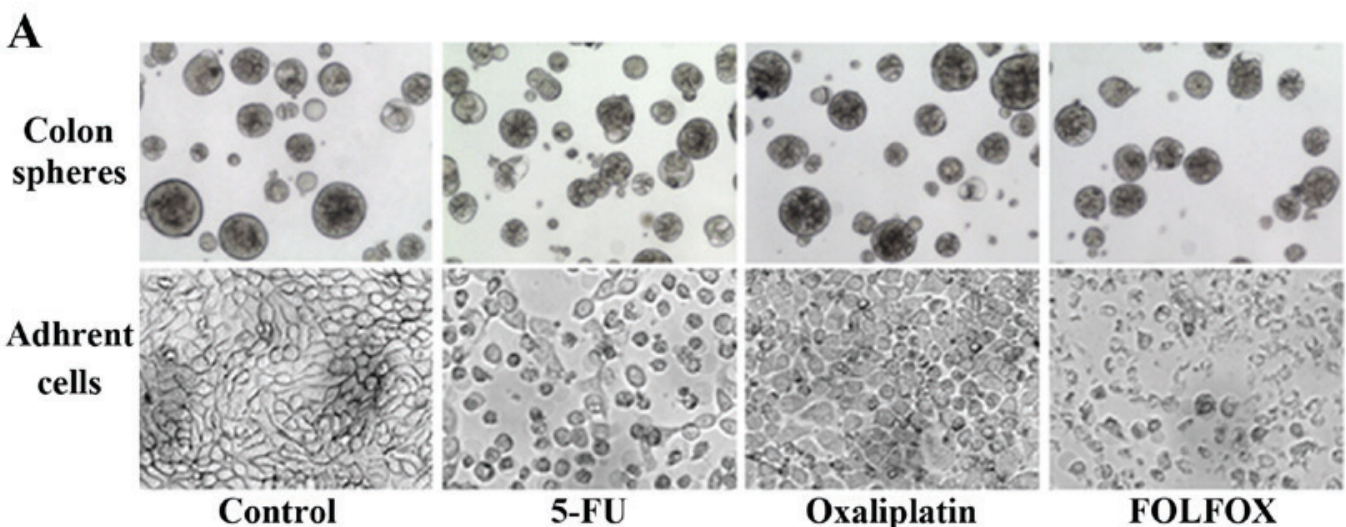

B
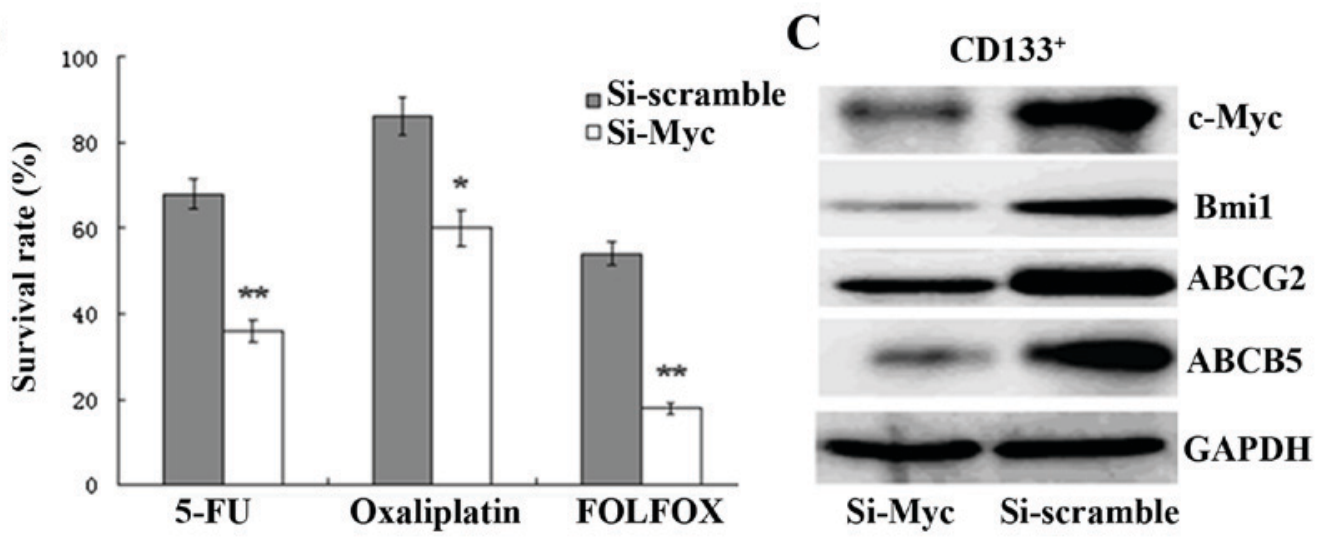

Figure 3. Depletion of c-Myc enhances the chemosensitivity of colon CSCs through the regulation of ABCG2 and ABCB5 expression. (A) Chemotherapy treatment of HT-29 adherent cells resulted in a significant increase in cell death and disintegration, compared with colon CSCs, as observed by inverted phase contrast microscopy (bars, $50 \mu \mathrm{m}$ ). (B) The survival rates of c-Myc-siRNA-transfected CD133+ cells were significantly reduced compared with the scramble-siRNA group following treatment with 5-FU, oxaliplatin or FOLFOX $\left({ }^{*} \mathrm{P}<0.05,{ }^{* *} \mathrm{P}<0.01\right)$. (C) c-Myc, Bmi1, ABCG-2 and ABCB5 were all downregulated in $\mathrm{CD}_{13} 3^{+}$colon $\mathrm{CSCs}$, as determined by western blotting, following treatment with c-Myc siRNA. siRNA, small interfering RNA; CSC, cancer stem cell; FOLFOX, 5-FU plus oxaliplatin.

High expression of the ATP-binding cassette and multidrug resistance protein is essential for CSC chemoresistance (15-18). In the present study, a strong decrease was found in ABCG2 and ABCB5 expression upon c-Myc silencing (Fig. 3C). The results show that $\mathrm{c}-\mathrm{Myc}$ silencing enhances the chemosensitivity of colon CSCs through the downregulation of ABCG2 and $\mathrm{ABCB} 5$ expression, thus representing a valid approach for sensitizing colon CSCs to conventional treatment.

\section{Discussion}

There is accumulating evidence supporting the fact that tumors contain a small subpopulation of CSCs, which have a self-renewing capacity and are responsible for tumor maintenance and metastasis. In colon cancer, CD133 is regarded as a specific marker for the isolation and identification of CSCs in primary colon cancer, and in colon cancer cell lines (3-9). In the present study, we purified CD133 ${ }^{+}$colon CSCs from HT-29 cell line by FACS. CD133 ${ }^{+}$cells have high expression of 'stemness' genes, including CD133, Sox2, Oct4, c-Myc and Wnt2; many of these molecular markers have been previously reported to be involved in the maintenance of stemness in human CSCs (19). Additionally, it was found that isolated CD133+ cells were able to generate tumor spheres when cultivated in serum-free stem cell medium supplemented with $20 \mathrm{ng} / \mathrm{ml} \mathrm{EGF}$ and $10 \mathrm{ng} / \mathrm{ml}$ FGF-2, which sustained the cells in an undifferentiated state as sphere-like cellular aggregates. In addition, it was determined that the secondary passage tumor spheres have a larger volume and a more compact sphere structure (data not shown), suggesting that they retained capacity for self-renewal. The results of the present study demonstrated that $\mathrm{CD} 133^{+}$cells are endowed with stem cell-like properties.

c-Myc has been proven as one of 4 major factors that render the reprogramming capability of adult cells into germline-competent-induced pluripotent stem cells, suggesting that c-Myc is an important regulator of stem cell biology (11). Previous studies showed that c-Myc expression may be implicated in tumorigenesis via activating its downstream target genes (10). These results suggested that c-Myc may serve as a link connecting malignancy and 'stemness'. Herein, it was also demonstrated that c-Myc expression was upregulated in $\mathrm{CD}_{133}{ }^{+}$colon CSCs (Fig. 1A). c-Myc expression was downregulated in $\mathrm{CD}_{133^{+}}$colon $\mathrm{CSC}$ by siRNA and examined to discern whether it would affect the cells. First, it was observed that treatment with c-Myc siRNA can block sphere formation (Fig. 2A) inhibit the invasion and migration potential of $\mathrm{CD}_{133^{+}}$colon CSCs in vitro (Fig. $2 \mathrm{~B}$ ). In addition, tumor growth in vivo was suppressed by c-Myc siRNA (Fig. 2C), and it was found that the Bmil, which controls many diverse biological cancer processes such as differentiation, proliferation, migration, and tumorigenesis (20), was downregulated in the c-Myc siRNA group (Fig. 3C). These data show that c-Myc 
is essential for survival, and that colon CSC self-renewal is consistent with the findings in brain CSCs (12).

Curative treatment failure in patients with cancer often occurs as a result of intrinsic or acquired resistance of tumors to chemotherapeutic agents (7). 5-FU, or 5-FU plus oxaliplatin (FOLFOX), which remains the backbone of colorectal cancer chemotherapeutics, still shows limited success (2). As previously reported, CSCs show resistance to conventional therapies, which may explain why it is difficult to completely eradicate cancer and why recurrence is an ever-present threat (7-9). Consistent with these hypotheses, colon cell spheres were significantly resistant to 5-FU and oxaliplatin compared with HT-29 adherent cells; even treatment combined with FOLFOX failed to cause death among treated colon cell spheres (Fig. 3A). Therapeutic strategies that specifically target colon CSCs are likely to be effective in eradicating tumors (21-24). To overcome the resistance to chemotherapy of colon CSCs, treatment with c-Myc siRNA was used, and the results showed that c-Myc knockdown can significantly improve the anti-cancer effects in single or combination-treated $\mathrm{CD}_{133}{ }^{+}$colon $\mathrm{CSCs}$ in vitro (Fig. 3B).

Understanding how chemoresistance develops, and eventually how it can be prevented, is crucial to fighting cancer effectively $(23,24)$. Recent studies have revealed that the $\mathrm{ABC}$ transporter family are involved in multidrug resistance (15-18). Xie et al (15) observed that 'side population' CSCs in colon cancer expressed high levels of ABCG2 and had a greater capacity to expel cytotoxic drugs. Furthermore, Porro et al (14) showed that c-Myc may contribute to the multidrug resistance profile and malignant progression of myeloid tumors by dysregulating the transcription of specific ABC transporter genes. Similarly, Kugimiya et al (18) reported that c-Myc confers resistance to 5-FU through regulating ABCB5 expression in human colon cancer cells. Accordingly, in the present study, it was found that $\mathrm{CD} 133^{+}$colon $\mathrm{CSCs}$ were highly co-expressed with ABCG2 and ABCB5 (data not shown). Furthermore, it was observed that there was a significant downregulation of ABCG2 and ABCB5 expression in $\mathrm{CD}_{133}{ }^{+}$colon CSCs after c-Myc siRNA treatment was administered (Fig. 3C), thus, enhancing the chemosensitivity of colon CSCs (Fig. 3B). These findings indicate that c-Myc silencing sensitizes colon CSCs to chemotherapy-induced cytotoxicity, at least in part via the downregulation of ABCG2 and $\mathrm{ABCB}$, although the molecular mechanisms remain to be fully clarified; our group is currently planning further study into this.

In conclusion, the data suggest that c-Myc knockdown suppresses the self-renewal, tumorigenicity, invasion and drug resistance of colon CSCs. c-Myc is, therefore, indispensable for the maintenance of colon CSCs, and targeting c-Myc may be an effective therapeutic strategy for eliminating colon cancer.

\section{Acknowledgements}

Not applicable.

\section{Funding}

The present study was supported by grants from the National Natural Science Foundation of China (no. 81372209), and the Project of Science and Technology of Ningbo City (nos. 2014A610223 and 2017A610153).

\section{Availability of data and materials}

The datasets used and/or analyzed during the present study are available from the corresponding author on reasonable request.

\section{Authors' contributions}

HLZ and PW performed all experiments in the present study. LZ and SDZ analyzed the data, and HLZ and MZL designed all experiments in the present study.

\section{Ethics approval and consent to participate}

The present study was approved by the Ethics Committee of Lihuili Hospital of Ningbo Medical Center (Ningbo, China).

\section{Patient consent for publication}

Not applicable.

\section{Competing interests}

The authors declare that they have no competing interests.

\section{References}

1. Siegel R, Ma J, Zou Z and Jemal A: Cancer statistics, 2014. CA Cancer J Clin 64: 9-29, 2014.

2. Thomassen I, van Gestel YR, Lemmens VE and de Hingh IH: Incidence, prognosis, and treatment options for patients with synchronous peritoneal carcinomatosis and liver metastases from colorectal origin. Dis Colon Rectum 56: 1373-1380, 2013.

3. Ieta K, Tanaka F, Haraguchi N, Kita Y, Sakashita H, Mimori K, Matsumoto T, Inoue H, Kuwano $\mathrm{H}$ and Mori M: Biological and genetic characteristics of tumor-initiating cells in colon cancer. Ann Surg Oncol 15: 638-648, 2008.

4. O'Brien CA, Pollett A, Gallinger S and Dick JE: A human colon cancer cell capable of initiating tumour growth in immunodeficient mice. Nature 445: 106-110, 2007.

5. Ricci-Vitiani L, Lombardi DG, Pilozzi E, Biffoni M, Todaro M, Peschle $\mathrm{C}$ and De Maria R: Identification and expansion of human colon-cancer-initiating cells. Nature 445: 111-115, 2007.

6. Zhang H, Li W, Nan F, Ren F, Wang H, Xu Y and Zhang F: MicroRNA expression profile of colon cancer stem-like cells in HT-29 adenocarcinoma cell line. Biochem Biophys Res Commun 404: 273-278, 2011.

7. Anderson EC, Hessman C, Levin TG, Monroe MM and Wong MH: The role of colorectal cancer stem cells in metastatic disease and therapeutic response. Cancers 3: 319-339, 2011.

8. Huang EH and Wicha MS: Colon cancer stem cells: Implications for prevention and therapy. Trends Mol Med 14: 503-509, 2008.

9. Fang DD, Kim YJ, Lee CN, Aggarwal S, McKinnon K, Mesmer D, Norton J, Birse CE, He T, Ruben SM, et al: Expansion of $\mathrm{CD}_{133^{+}}$colon cancer cultures retaining stem cell properties to enable cancer stem cell target discovery. Br J Cancer 102: 1265-1275, 2010.

10. Meyer N and Penn LZ: Reflecting on 25 years with MYC. Nat Rev Cancer 8: 976-990, 2008.

11. Takahashi K and Yamanaka S: Induction of pluripotent stem cells from mouse embryonic and adult fibroblast cultures by defined factors. Cell 126: 663-676, 2006.

12. Wang J, Wang H, Li Z, Wu Q, Lathia JD, McLendon RE, Hjelmeland $\mathrm{AB}$ and Rich JN: c-Myc is required for maintenance of glioma cancer stem cells. PLoS One 3: e3769, 2008. 
13. Porro A, Haber M, Diolaiti D, Iraci N, Henderson M, Gherardi S Valli E, Munoz MA, Xue C, Flemming C, et al: Direct and coordinate regulation of ATP-binding cassette transporter genes by Myc factors generates specific transcription signatures that significantly affect the chemoresistance phenotype of cancer cells. J Biol Chem 285: 19532-19543, 2010.

14. Porro A, Iraci N, Soverini S, Diolaiti D, Gherardi S, Terragna C, Durante S, Valli E, Kalebic T, Bernardoni R, et al: c-MYC oncoprotein dictates transcriptional profiles of ATP-binding cassette transporter genes in chronic myelogenous leukemia CD34 hematopoietic progenitor cells. Mol Cancer Res 9: 1054-1066, 2011.

15. Xie ZY,Lv K, Xiong Y and Guo WH: ABCG2-meditated multidrug resistance and tumor-initiating capacity of side population cells from colon cancer. Oncol Res Treat 37: 666-668, 670-672, 2014.

16. Tang Y, Hou J, Li G, Song Z, Li X, Yang C, Liu W, Hu Y and $\mathrm{Xu} \mathrm{Y}$ : ABCG2 regulates the pattern of self-renewing divisions in cisplatin-resistant non-small cell lung cancer cell lines. Oncol Rep 32: 2168-2174, 2014.

17. Xie N, Mou L, Yuan J, Liu W, Deng T, Li Z, Jing Y and Hu Z: Modulating drug resistance by targeting $B C R P / A B C G 2$ using retrovirus-mediated RNA interference. PLoS One 9: e103463, 2014.

18. Kugimiya N, Nishimoto A, Hosoyama T, Ueno K, Enoki T, Li TS and Hamano K: The c-MYC-ABCB5 axis has a pivotal role in 5 -fluorouracil resistance in human colon cancer cells. J Cell Mol Med 19: 1569-1581, 2015.

19. Amini S, Fathi F, Mobalegi J, Sofimajidpour H and Ghadimi T: The expressions of stem cell markers: Oct4, Nanog, Sox2, nucleostemin, Bmi, Zfx, Tcl1, Tbx3, Dppa4, and Esrrb in bladder, colon, and prostate cancer, and certain cancer cell lines. Anat Cell Biol 47: 1-11, 2014.
20. Cao L, Bombard J, Cintron K, Sheedy J, Weetall ML and Davis TW: BMI1 as a novel target for drug discovery in cancer. J Cell Biochem 112: 2729-2741, 2011.

21. James MI, Iwuji C, Irving G, Karmokar A, Higgins JA, Griffin-Teal N, Thomas A, Greaves P, Cai H, Patel SR, et al: Curcumin inhibits cancer stem cell phenotypes in ex vivo models of colorectal liver metastases, and is clinically safe and tolerable in combination with FOLFOX chemotherapy. Cancer Lett 364: 135-141, 2015.

22. Todaro M, Alea MP, Di Stefano AB, Cammareri P, Vermeulen L, Iovino F, Tripodo C, Russo A, Gulotta G, Medema JP, et al: Colon cancer stem cells dictate tumor growth and resist cell death by production of interleukin-4. Cell Stem Cell 1: 389-402, 2007.

23. Kreso A, van Galen P, Pedley NM, Lima-Fernandes E, Frelin C, Davis T, Cao L, Baiazitov R, Du W, Sydorenko N, et al: Self-renewal as a therapeutic target in human colorectal cancer. Nat Med 20: 29-36, 2014.

24. Zeuner A, Todaro M, Stassi G and De Maria R: Colorectal cancer stem cells: From the crypt to the clinic. Cell Stem Cell 15: 692-705, 2014.

This work is licensed under a Creative Commons Attribution-NonCommercial-NoDerivatives 4.0 International (CC BY-NC-ND 4.0) License. 